\title{
FINITE ELEMENT ANALYSIS OF TEMPERATURE DISTRIBUTION IN SPORT HORSES' SKIN DURING REHABILITATION MASSAGE
}

\author{
Roman Trochimczuk, Piotr Prochor \\ Bialystok University of Technology, Poland \\ r.trochimczuk@pb.edu.pl, piotrekprochor@gmail.com
}

\begin{abstract}
High physical strain placed on sport horses forces breeders to search for new methods and rehabilitation devices in order to restore animals to full, proper activity. One of the currently used method is a manual massage of the animal performed for both - the rehabilitation and relaxation purposes before, during and after physical activities. Because of the time needed to perform the massage, the difficulty of carrying it out by a physiotherapist, as well as the measurements of horse-patients, such procedures are supported by various mechanical systems. For this reason, currently numerous studies are carried out, which goal is to find optimal solutions that increase the quality of the animal care. The results of these researches reduce the time to service a single horse staying in a stud. The following paper presents the numerical studies, conducted in the ANSYS Workbench v.16.2 software, which describe the temperature distribution in the skin of sport horses during the rehabilitation massage with exemplary technical massaging system. Two variable parameters for the analyses were considered: the speed of the massager tip and the coefficient of friction between the material of the massager tip and the skin of the animal. Temperature generated in the skin during the rehabilitation and relaxation massage has a significant impact on the effectiveness and speed of the restoration to the appropriate functionality of the sport horses. It also helps in maintaining a good mental and physical condition of the animal and shortens the recovery time after strenuous athletic training. Thus, it increases the willingness to take on new challenges. Additionally, the directions of further research are indicated in the conclusions of the article.
\end{abstract}

Keywords: temperature distribution, FE analysis, ANSYS, skin modeling, rehabilitation massage, sport horse.

\section{Introduction}

Effective breeding of sport horses force stable owners to search for new as well as more and more efficient solutions that contribute to improvement of animals' living conditions. Modern technical systems supporting daily activities of animals, their training, relaxation and rehabilitation (before, after and during training), have already become a standard or even a necessary equipment of any professional breeder. The high price that has to be covered in order to train a sport horse and make it ready to take a challenge and a risk associated with it, ultimately translates to high economic value of a specific animal. Depending on the horse's breed and its destination, it is necessary to provide an individually adjusted, balanced diet, appropriate living conditions (i.e. the type of stable; jogging track; the ability to provide comfort, freedom and safety in horsebox; a proper walking surface; daily hygiene and daily care) and to adjust the type and intensity of the workout [1]. Because of the extreme effort that horses put in the highly demanding training, or even due to unadjusted diet, the horse's temper, poor training conditions or owner's incompetence, sport horses often suffer various kinds of injuries. For this reason, malaise is often observed that may lead to jabbering. It demotivates the horse and, in the worst cases, makes competing impossible. All these factors increase a possibility of developing diseases (including different kinds of bone and/or muscle pain) and the lack of energy as well as the desire for further training with its rider.

Apart from the typical veterinary care, appropriate breeding and carefully planned training, as in the case of human athletes, are supported with appropriate massage and physiotherapy treatment. Among others these treatments include: hydrotherapy (e.g. water treadmill; aquatic spa - the impact of cold or heat on animal's body; jacuzzi that allows to perform water massage process; swimming pool), solarium, magnetic and electromagnetic rehabilitation systems, oxygen therapy, chiropractic, acupuncture and manual massage supported with appropriate technical systems [1-3].

Manual massage of horses is currently used mainly for rehabilitation but it finds its use also in after-training relaxation process. The method is gaining popularity as it is one of the simplest forms of physiotherapy - it can be a form of stroking, kneading, clapping or grinding. Typically, masseurs use only the power of their hands, sometimes supporting their work with the use of, e.g., appropriate technical devices which produce additional vibrations. Due to the fact that the horse body has a substantial size and the skin is of a significant thickness, the physical realisation of the massage process is time-consuming and labour-intensive even for experienced animals' masseurs [2;4]. 
Properly executed massage process requires from the horses' physiotherapist a specialist knowledge about the anatomy of the animal. With its regular use a rigorous training schedules can be maintained, which can reduce or remove the negative side-effects arising from intense exercises. As a result, it increases the potential to restore appropriate motor conditions and maximum use of the capabilities of an animal. A proper rehabilitation massage helps to relieve muscle tension (increasing their flexibility), increases range of motion (by stretching the tendons and ligaments), improves blood and lymph circulation in the body and consequently increases the strength of the body, preventing future injuries. Temperature arising during massage causes the expansion of blood vessels, stimulating and nourishing tissues to greater metabolic activity. This accelerated blood flow, by relaxing and reducing tension, increases muscle flexibility while reducing pain after exercises, among others, caused by lactic acid production.

The technical solution of rehabilitation massage system proposed in this paper is a response to the needs identified by breeders, trainers and horses' masseurs, resulting mostly from the need of easing the daily care fatigue. The following paper presents the numerical studies conducted in the ANSYS Workbench v.16.2 software, which describe the temperature distribution in the skin of sport horses during the rehabilitation massage with exemplary technical massaging system. The temperature generated in the skin during the rehabilitation and relaxation massage has a significant impact on the effectiveness and speed of recovering the appropriate functionality of the sport horses.

\section{Materials and methods}

Based on the developed invention, reported to the Patent Office of the Republic of Poland [5], concerning a mechatronic device for lymphatic drainage of human upper and lower limbs, the authors proposed a new solution, which after minor changes from the original design, can be adapted to the rehabilitation of sport horses. This device is mounted on the animal's body with the use of special belts and straps that maintain a proper position of the device's base - Fig. 1A, detail (1). Its final form cannot be presented in detail in this paper due to the activities related to the patenting process. In the case discussed for the purposes of this study, it is assumed that a massage treatment (rehabilitation) and/or relaxation, will be carried out in the area of the horse's wither (A), the back (B) reaching the croup (C), and it will be performed with linear moves - Fig. 1A, detail (2). The main difference in comparison with the original design concerns a method of individually adjusting the device to the zoometric parameters of the animal. In the new solution, instead of the conventional linear guide with adjustable height and the intensity of pressure on the skin, longer, arc-shaped guides were applied that can be easily adjusted to the overall dimensions of individual animal - Fig. 1B, detail (3). Their form and length depends on the place of massage. Another fundamental change from the original design is the size of massage end-effectors - Fig. 1B, the detail (6) mounted on a trolley track (5). Motion of the trolley track (5) with single massage end-effector (6), is forced by a tie trolley (4) connected with the drive. The quantity of curvilinear guides (3) depends on the area of massage. Their length has proper limits due to the necessity to ensure free movement on the rail curvature.
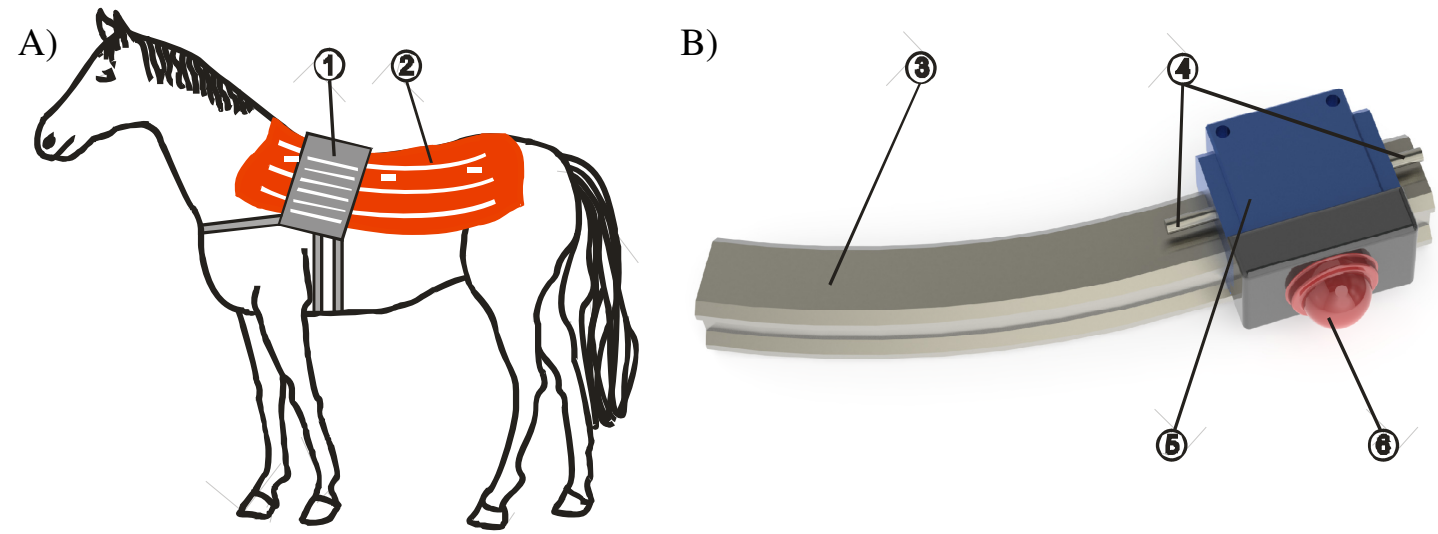

Fig. 1. A) Example of using the proposed massage system to rehabilitation of the skin of the horse's back, B) single curvilinear guide with massage end-effector: 1 - rehabilitation system; 2 - massage area; 3 - curvilinear guide; 4 - tie trolley; 5 - trolley track; 6 - single massage end-effector 
The possibility of changing the characteristics of the operating modes (i.e. changing the immersion depth of the single massage end-effector, controlling the massaging speed or the option to set additional vibration on effectors) remains the same as in the original.

In order to analyse how temperature is generated in horse's skin during the rehabilitation massage, calculations with the use of the finite element method were conducted in ANSYS Workbench software v.16.2 (ANSYS Inc.). The presented analyses include a simplified model of the skin by merging each of its individual layers. This simplification is possible due to the fact that the mechanical and thermal properties of the individual layers differ only slightly from each other. This has been used in various studies that analyse human skin, among others in Çetingül and Herman research [6]. However, the chemical composition of the skin is similar in mammals [7], which allows the use of this simplification in analysing the horse's skin.

The studies analysed the massage process conducted with the use of a single, non-rotatable massage end-effector in the shape of a hemisphere. To simulate a massage an appropriate motion of massage end-effector was included. Overall dimensions of the simplified skin model and the massage end-effector are shown in Figure 2A. The trajectory of the massage end-effector incorporated in the study is given in Figure 2B. An appropriate massage cycle was adopted for the purpose of the numerical model. The massage begins with immersion of the single massage end-effector to $1.5 \mathrm{~mm}$ and then it continues with linear displacement of $15.0 \mathrm{~mm}$ with established speed. In the next phase of the cycle, the immersion is decreased by $1.0 \mathrm{~mm}$ and the end-effector is moved, in the opposite direction to the primordial direction, over the skin at a distance of $30.0 \mathrm{~mm}$ (the idle phase relaxation). It is followed by re-immersion of the end-effector on by $1.0 \mathrm{~mm}$ (1.5 $\mathrm{mm}$ total immersion) and linear feed rate on the distance of $30.0 \mathrm{~mm}$ (in primordial direction). Then again a relaxation phase is performed, which is followed by appropriate linear feed rate. The process is repeated until the end of the massage. In the model a variable skin thickness, from $4.4 \mathrm{~mm}$ to $3.0 \mathrm{~mm}$, was considered, which is a characteristic feature of the horse's ridge skin [8], that is the most commonly massaged place. This allows for a better reflection of the actual, anatomical conditions.

A)

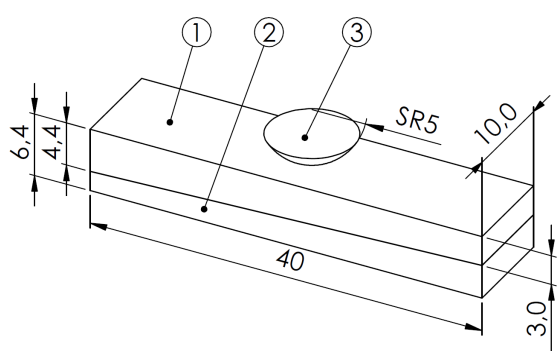

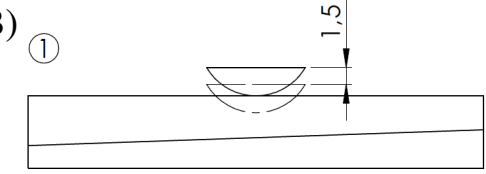

(2)

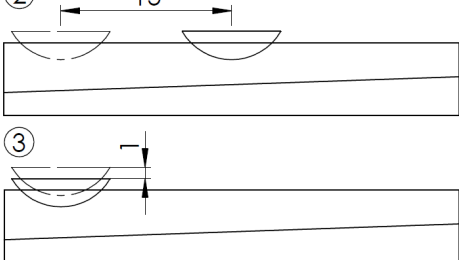

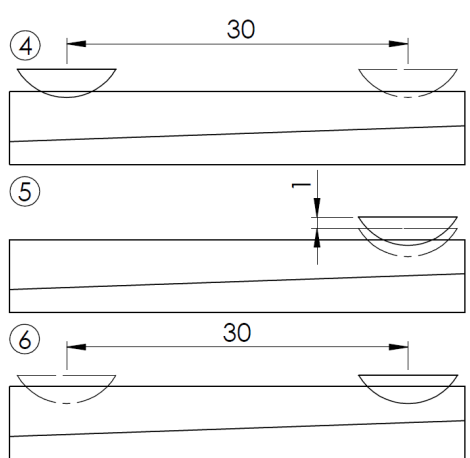

Fig. 2 A) Overall dimensions of the simplified model of the skin and the hemispherical massage end-effector: 1 - skin; 2 - tissues under skin; 3 - hemispherical massage end-effector. B) the pattern of hemispherical massage end-effector motion: 1 - immersion; 2 - initial massage; 3 -reduction of the immersion; 4 - idle phase - relaxation; 5 - re-immersion; 6 - feed rate - massage phase (in $\mathrm{mm}$ )

Discretisation of the model was performed using a 10-node solid227 element [9]. The size of the elements has been optimised with the use of the mesh refinement test with $5 \%$ convergence error for von-Mises stress maximal values, which resulted in obtaining a model consisting of 9413 elements. In the analyses the isotropic mechanical and thermal properties of the skin as well as appropriate physical properties were used: density $=1200 \mathrm{~kg} \mathrm{~m}^{-3}$ [10]; Young's modulus $=0.5 \mathrm{MPa}$ [11]; Poisson's ratio is 0.5 [11]; Thermal conductivity $0.45 \mathrm{~W}^{-1} \mathrm{~K}^{-1}$ [10]; Specific heat $\mathrm{J}=2500 \mathrm{~kg}^{-1} \mathrm{~K}^{-1}$ [12]. The duration of the massage was set for 15 seconds. The effects of two variables on the temperature generated in the horse's skin during massage were analysed: the coefficient of friction and velocity of the massage end-effector. For the purpose of analyses the following values were selected: coefficient of friction is 0.8 and 0.9 (characteristic for the contact of skin with plastic [13]) and velocity of the massage endeffector $=30 \mathrm{mms}^{-1}$ and $40 \mathrm{~mm}^{-1}$. To take into account the reciprocal interaction between the massage end-effector and the skin, appropriate contact elements were applied [9]. The Gaussian 
method was used as a point detection method. Additionally, the pure penalty method was used to properly consider immersion of the massage end-effector into skin. To consider appropriate boundary conditions a fixed support was set on the bottom surface of tissues under skin. Tissues under skin (for the sake of analyses considered with the same properties as skin) were set, in order to allow the analysed skin to be displaced in its whole volume, as it is in actual conditions. The initial skin temperature was set as $38^{\circ} \mathrm{C}$ [14], while the ambient temperature as $24^{\circ} \mathrm{C}$, which properly reflects the conditions of a typical stable. The material for single massage end-effector was set as polyethylene. Its thermal and mechanical properties were taken from the ANSYS database.

\section{Results and discussion}

A number of results were received from the conducted numerical simulations. On their basis graphs were created, that present the effect of changes in velocity of the single massage end-effector or changes in the coefficient of friction on the temperature generated in skin of a rehabilitated sport horse (Fig. 3).

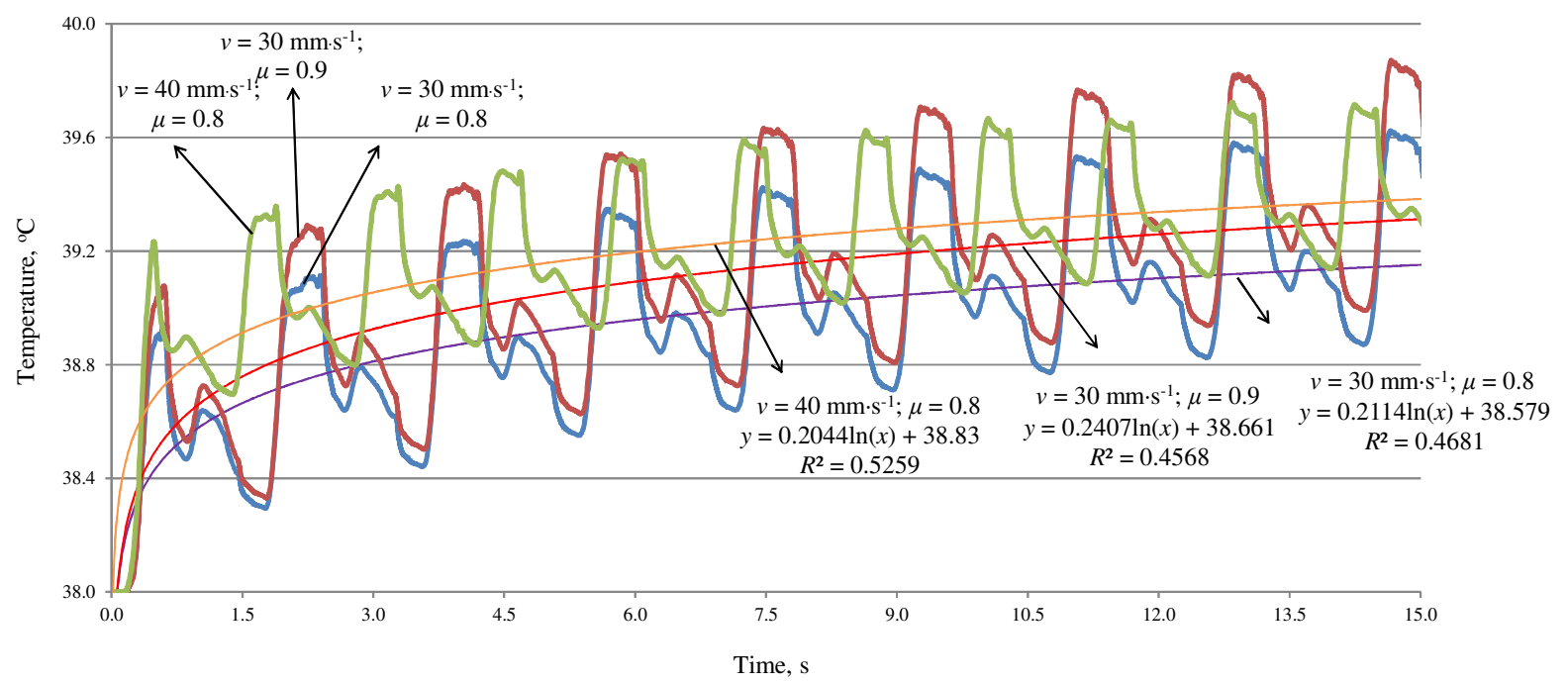

Fig. 3. Temperature distribution in horses' skin during the rehabilitation massage

A)

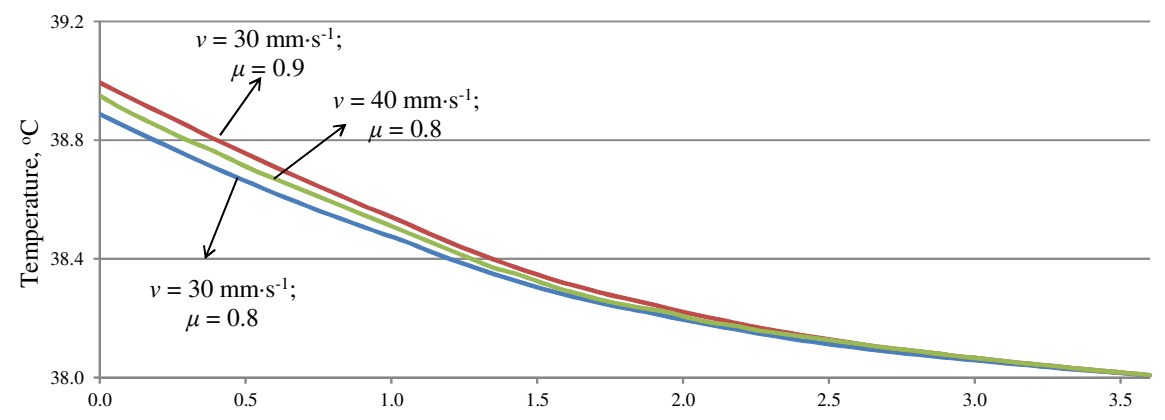

B)

Skin depth, mm

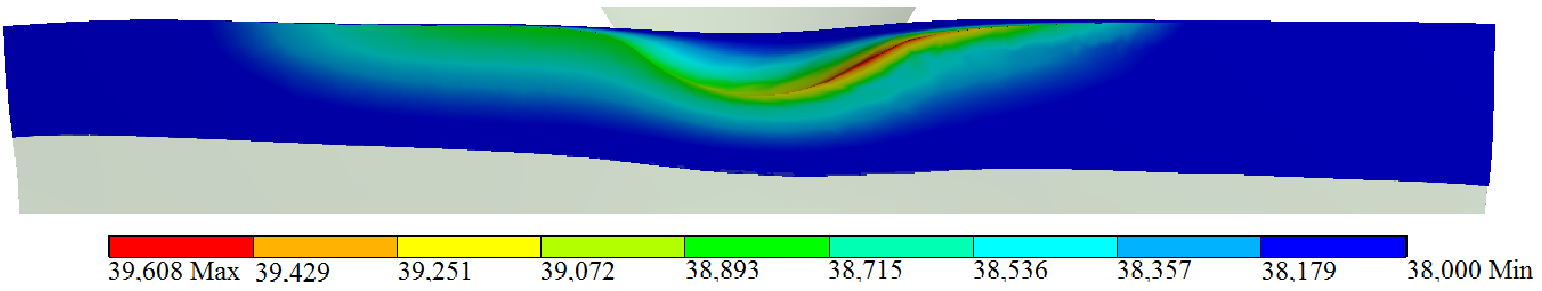

Fig. 4. A) Temperature distribution at certain depths of the horse's skin (measured in the middle

part of the model during last, calculated cycle); B) cross-section of an example of a map of temperature generated on the horse's skin in $15 \mathrm{sec}$. of simulation $\left(v=30 \mathrm{~mm}^{-1}\right.$ and $\left.\mu=0.8\right)$

For the obtained results, appropriate logarithmic regression curves were created, with the use of MS EXCEL 2010 (Microsoft Corp.), allowing the long-term estimation of the analysed process. Fig. 4A contains a graph that presents the temperature at certain depths of the skin (measured in the 
middle part of the model during the last, calculated cycle), while Fig. 4B shows an example of a map of temperature generated on the horse's skin in $15 \mathrm{sec}$. of simulation.

Analysing the obtained results allowed to conclude:

1. The highest temperature rises during the massage phase and is observed at the end of the massage phase with immersion of $1.5 \mathrm{~mm}$. This increase equals approx. $0.25^{\circ} \mathrm{C}$ for one cycle. In the idle phase (relaxation phase) at immersion of $0.5 \mathrm{~mm}$ the temperature is maintained. Its change equals approx. $0.025^{\circ} \mathrm{C}$ during one cycle. The total temperature change during 15 seconds simulation is approx. $1.4^{\circ} \mathrm{C}$.

2. With the end-effector velocity of $30 \mathrm{mms}^{-1}$, the temperature generated in the horse's skin is lower than at the higher speed of $40 \mathrm{~mm}^{-1}$. A higher number of cycles allows for faster accumulation of heat produced in the skin and reduces the relaxation phase (cooling the horse's body).

3. The selection of the end-effector's material with a higher coefficient of friction, in relation to the skin, increases the temperature generated during the massage. The increase for a single cycle equals approx. $0.25{ }^{\circ} \mathrm{C}$ for the end-effector velocity of $30 \mathrm{~mm}^{-1}$ and $0.2^{\circ} \mathrm{C}$ for the end-effector velocity of $40 \mathrm{~mm} \mathrm{~s}^{-1}$.

\section{Conclusions}

1. Performing the massage manually, without the help provided by a technical system, is quite problematic and exhaustive to animal physiotherapist, among others due to the size of the animal. The innovative technical solution proposed in the presented paper can be used simultaneously in different places on the body of the animal. Depending on the needs, individual systems can work in the same or different massage modes (e.g., one system performs typical rehabilitation massage, while another executes relaxation massage).

2. The use of numerical methods to estimate the temperature distribution generated during the massage of the horse's skin shortens the work on a laboratory prototype device and allows to properly adapt the motion control strategies of massage end-effectors. With the obtained results from the finite element analyses, appropriate operating modes can be developed, which can be individually used as the relaxation or rehabilitation massage process.

3. The coefficient of friction that characterises the material of the massage end-effector influences the rise in temperature generated in the skin during the massage. Thus, the device prototype considers using different materials according to the patient's specific needs and the type of the massage.

4. The size of the hemispherical end-effector used for the presented numerical research corresponds to the form and size of manual, commercial massagers that are used among others in special curry combs. The use of multiple end-effectors affecting the animal's body at the same time, allows to increase the intensity of the performed massage.

5. Immersion while the massage phase should be greater than in the relaxation phase. This is because the massage end-effector motion should correspond to the manual massage performed by a qualified physiotherapist. However, this value must be chosen rationally, as too high immersion can cause discomfort of the rehabilitated animal instead of a therapeutic effect. Properly adjusted value apart from heat generated during massage, influences proper lymph flow in the massaged part of horse's body at the same time, which has a significant effect in relaxing tissues after intense trainings.

6. The temperature generated during the massage of the horse's skin causes vasodilation. As a result, the tissues are stimulated to greater metabolic activity and are more nourished, which fastens regenerative processes of the body. Therefore, the knowledge of the mechanisms of temperature generation and cooling processes during massage with the use of a proper end-effector allows for appropriate selection of the parameters and positioning of the device. This, above all, includes the velocity and the proper selection of acceleration that provide movement of the end-effector along the curvilinear guide, which will not cause any discomfort during the use of the rehabilitation system.

7. The proper rehabilitation time of an animal usually lasts approx. 15 minutes. Adopted, for the simulation purposes, massage time of 15 seconds was intentionally chosen because of the time needed to perform numerical calculations with the use of the finite element method. However, the 
obtained results give a general idea over the physics of the process of generating the temperatures in the skin of a rehabilitated sport horse, both during the massage and idle phases. For this reason, the logarithmic regression curves with appropriate equations were calculated in order to provide the possibility to estimate the temperature that can be obtained at any time of the massage process.

8. The increase of single massage end-effector velocity, while maintaining the coefficient of friction unchanged, has a major influence on the temperature created in the animal's body.

9. Significant impact on the temperature generated during the massage has the immersion of the single massage end-effector during both massage and relaxation phases. The higher value of the immersion results in generating higher temperatures.

10. Increasing the velocity of the massage end-effector or applying the material with a higher coefficient of friction in relation to the skin increases the temperature not only on the outer layer of the skin but also in its whole volume.

11. In future work on the prototype of the rehabilitation massage system there are planned additional researches to determine the optimal parameters for positioning end-effectors for different modes of the massage. Their results will also determine the impact of the shape and size of the endeffector on the massage process. In the testing phase of the prototype it is planned to use thermography to help in adapting the parameters of the different operating modes.

\section{Acknowledgement}

This work was performed within the framework of statutory work of the Department of Automatic Control and Robotics and Department of Biocybernetics and Biomedical Engineering at Bialystok University of Technology no. S/WM/1/2016, no. MB/WM/17/16 and financed with funds for scientific research from the Ministry of Science and Higher Education.

\section{References}

1. Waszkiewicz M. Modern methods used in rehabilitation of sport horses. Engineering Thesis, Warsaw University of Life Science - SGGW, Warsaw, 2007.

2. Soroko M. Skuteczność masażu leczniczego u koni (Benefits of equine massage therapy). Życie Weterynaryjne, vol. 88(1), 2013, pp. 44-46 (in Polish).

3. Lewandowski B., Olinski M., Wudarczyk S., Gronowicz A. A conceptual project of a device for human wrist functional rehabilitation. International Journal of Applied Mechanics and Engineering. 2016, vol. 21(4), pp. 923-932.

4. McBride S.D., A. Hemmings K., Robinson K. A preliminary study on the effect of massage to reduce stress in the horse. Journal of Equine Veterinary Science, vol. 24(2), 2004, pp. 76-81.

5. Patent application no. P.411811: Mechatroniczne urządzenie do drenażu limfatycznego (Mechatronic device for lymphatic drainage). Patent Office of the Republic of Poland (in Polish).

6. Çetingül M.P., Herman C. Quantification of the thermal signature of a melanoma lesion. International Journal of Thermal Sciences, vol. 50(4), 2011, pp. 421-431.

7. Montagna W. The structure and function of skin. Elsevier, 2012.

8. Volkering M.E. Variation of skin thickness over the equine body and the correlation between skin fold measurement and actual skin thickness. Doctoral thesis, Faculty of Veterinary Medicine Theses, Utrecht University Repository, Utrecht, 2009.

9. ANSYS Inc. ANSYS Mechanical APDL Element Reference Release 15.0. 2013.

10. Jiang S.C., Ma N., Li H.J., Zhang X.X. Effects of thermal properties and geometrical dimensions on skin burn injuries. Burns. vol. 28(8), 2002, pp. 713-717.

11. Magnenat-Thalmann N., Kalra P., Lévêque J.L., Bazin R., Batisse D., Querleux B. A computational skin model: fold and wrinkle formation. IEEE Transactions on Information Technology in Biomedicine, vol. 6(4), 2002, pp. 317-323.

12. Morgan K.G., Nyman G., Funkquist P. Heat balance in trotters during intense exercise. Nutrition of the exercising horse, EAAP publication, 2008, pp. 163-166.

13. Sivamani R.K., Goodman J., Gitis N.V., Maibach H.I. Friction coefficient of skin in real-time. Skin Research and Technology, vol. 9(3), 2003, pp. 235-239.

14. Green A.R., Gates R.S., Lawrence L.M. Measurement of horse core body temperature. Journal of Thermal Biology, vol. 30(5), 2005, pp. 370-377. 